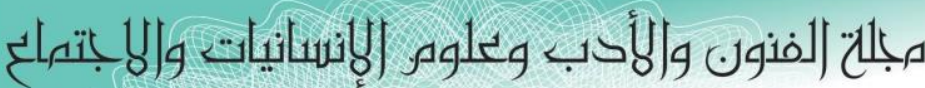

Journal of Arts, Literature, Humanities and Social Sciences

ISSN online: 2414 - 3383

ISSN print: 2616 - 3810

العدد (40) تهوز - يوليو 2019

\title{
The Roles of Vitamin D and Dietary Calcium in Nutritional Rickets
}

\section{Dr.Thamer Mahdi Saleh}

F.I.C.M.S ortho

Al Karkh General Hospital

Iraq

\author{
Dr. Saadi Hameed Abbood \\ .F.I.C.M.S Ortho \\ Al Karkh General Hospital \\ Iraq
}

\author{
Dr.Ahmed Shakir turky Al \\ awadi \\ .Diploma.ortho \\ Al Karkh General Hospital \\ Iraq
}

\begin{abstract}
The etiology and pathogenesis of nutritional rickets are becoming progressively clearer. Vitamin D deficiency has generally been considered the major or only player in the pathogenesis of nutritional rickets. However, recent research into calcium deficiency has now provided clinicians with reasons to investigate and manage patients with nutritional rickets more appropriately.

The important question when assessing cases of nutritional rickets is: "Is it calcium or vitamin $\mathrm{D}$ deficiency or both that play a major role in the pathogenesis of the disease"?

The case presentation in this review highlights the risk factors, clinical presentation and pathophysiology of nutritional rickets in a young in Baghdad, a city with abundant sunshine throughout the year.
\end{abstract}




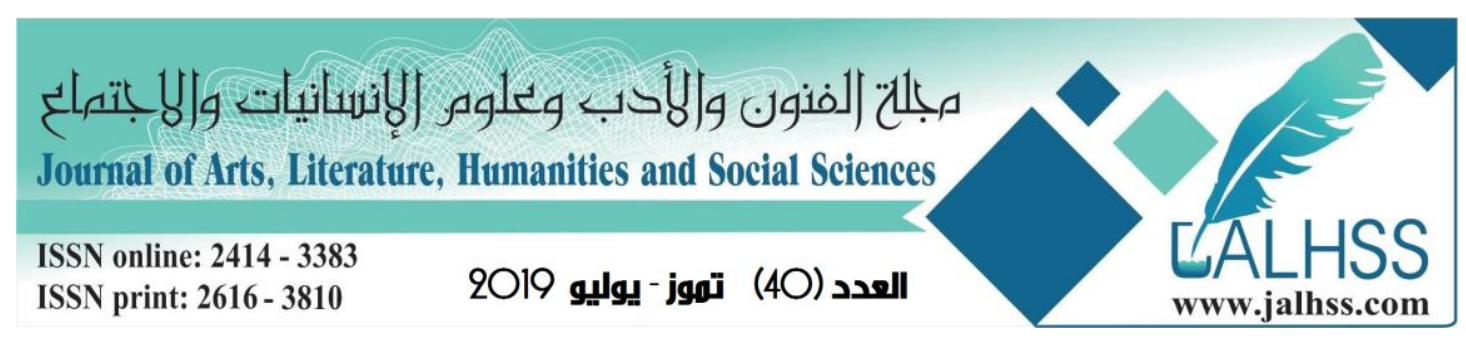

Case study: Case study: The patient, a 4 year old (Fig. 1), . The initial presenting complaint from the parents was that they had no- ticed that the child had an "odd" or "different" walking pat- tern over the previous year. He had started walking late at the age of 2 years, although cognitive development was normal. The parents had also noted deformities of the lower limbs from 2 and half years of age, which they described as an "odd shape of his legs". He had also become increasingly tired. The family is from a low socioeconomic community. When the child was still an infant, their home had burnt down and the young child had been placed in a children's home, while the parents re-established their lives. During this time, the child spent most of his time indoors. He was born by a normal vertex delivery with a birth weight $3.3 \mathrm{~kg}$. The infant had not been breastfed but did receive vitamin $\mathrm{D}$ fortified formula feeds up to 6 months of age. On ceasing formula feeds, his diet on history was considered to be poor with negligible amounts of milk and dairy products (total dietary calcium intake esti- mated to be $<200 \mathrm{mg} /$ day), and no red meat.

Our patient presented with stunting (height for age $\mathrm{Z}$ score $=-3.65$ ) and normal weight for height $(Z$ score $=0.98)$. He had a valgus deformity of the knees, but did not have widened wrists or a rachitic rosary. He did have a prominent forehead and reversal of the carrying angle of the elbows.

Based on the history, presenting complaints and clinical fea- tures, he was considered to have rickets, due most likely to nu- tritional causes, although hypophosphataemic rickets and skeletal dysplasias were considered as other unlikely possibilities.

The radiological findings are shown in Figs. 2-4. The salient findings were those of sclerotic bands at the distal ends of the metaphyses of the radius and ulna. Growth plates were widened, and a deformity was present at the distal end of the right radius possibly due to an old healed fracture. Lateral bowing of the right femur and valgus deformity of the left knee were noted. A possible old healed fracture of the right midshaft femur was also present. There were no metaphyseal irregularities at the proximal ends of the tibias or widened growth plates, however there were mild irregularities and slightly widened growth plates at the medial and lateral as- pects of the right and left distal ends of femurs respectively. The Thacher rickets severity score was assessed as being 1 .

Biochemical investigations were performed to establish whether or not rickets was present and if so the etiology of the disease, particular to determine if it was secondary to vitamin D or dietary calcium deficiency (Table 1). Based on the biochemical findings, the patient was commenced on vitamin D and calcium carbonate therapy.

\section{Introduction}

Nutritional rickets remains a global public health concern, parti- cularly in a number of lower-middle income countries and among at-risk immigrant communities in resource rich countries, as has been highlighted by a recent international consensus statement, despite a greater understanding of the etiology and pa- thogenesis of the condition. Vitamin D deficiency has generally been considered to be the primary cause of nutritional rickets, however more recent research into dietary calcium 


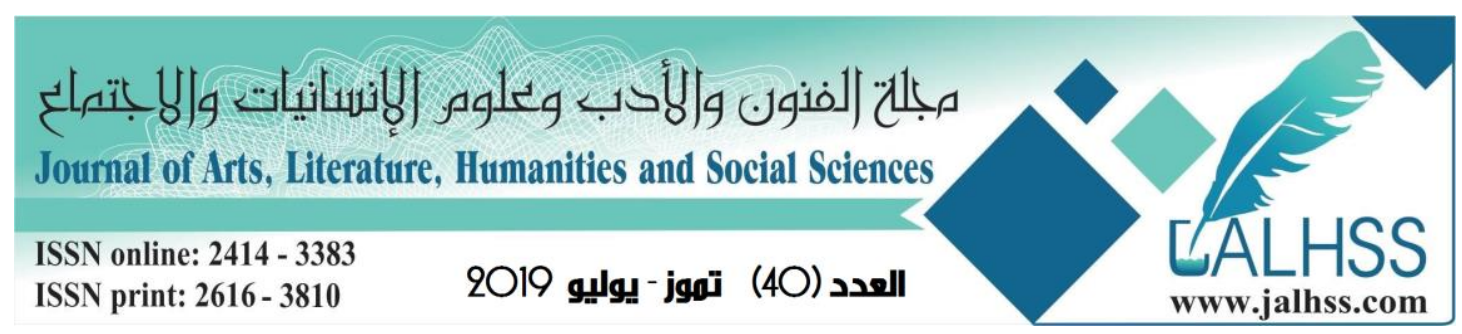

deficiency has now provided clinicians with reasons to investigate and manage patients with nutritional rickets differently. Although vitamin D deficiency is probably the commonest cause of nutritional rickets globally, dietary calcium deficiency has been identified as a common cause of nutritional rickets in a number of developing countries .

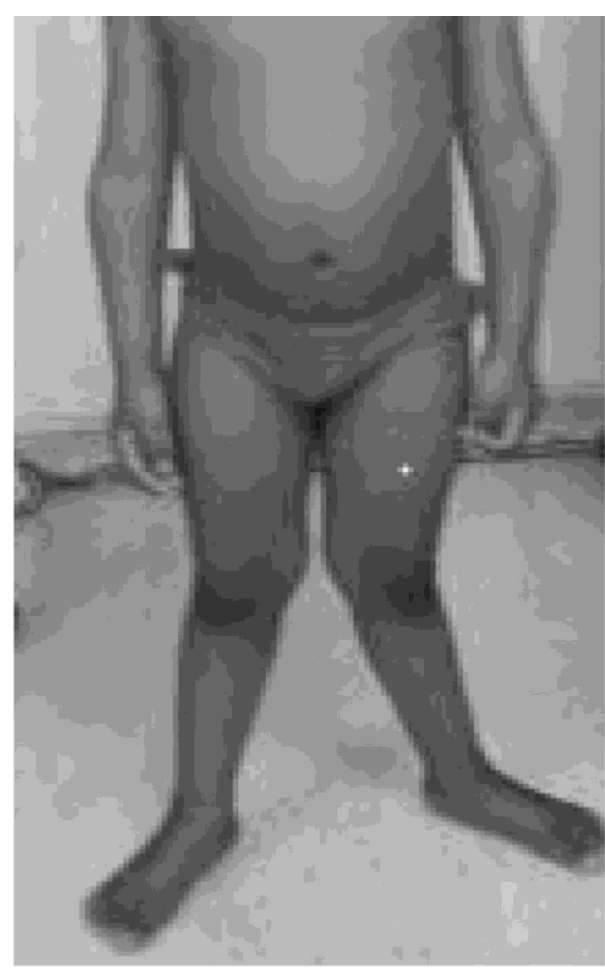

Fig. 1. The index patient presented with genu valgus deformities of the lower limbs at the age of 4 years.

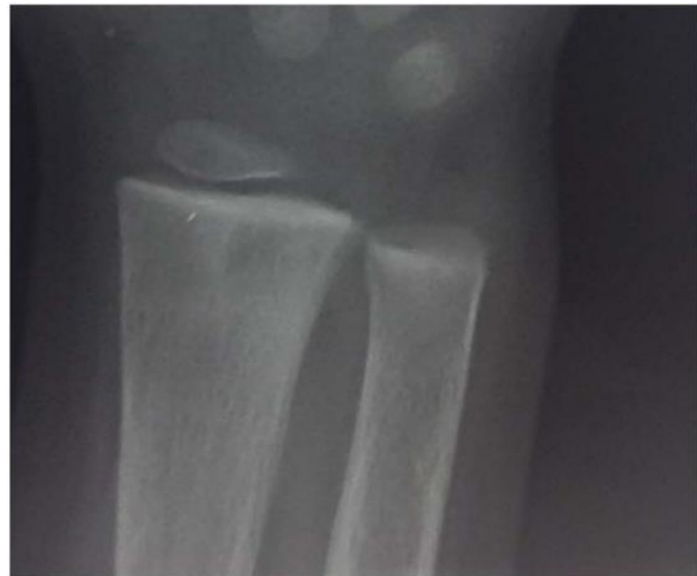

A

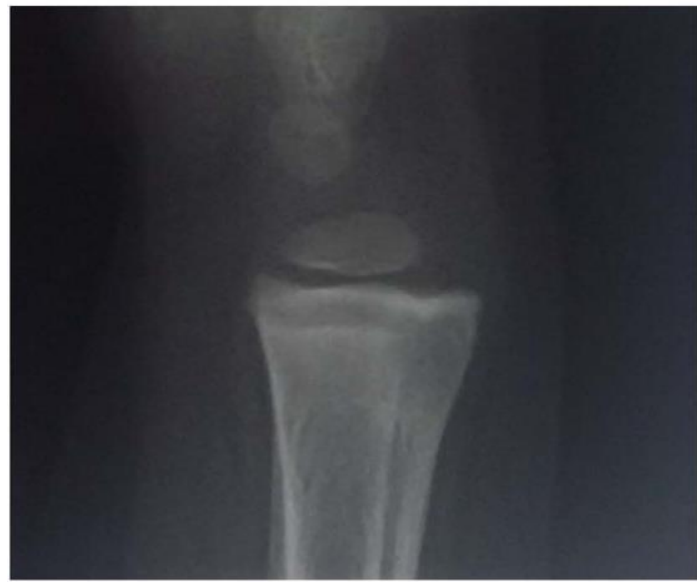

Fig. 2. A and B: X-ray of right wrist (AP and lateral views) showing sclerotic bands of healing at the distal metaphyses and possible healed distal radius fracture (evidenced by remodeling defect (tubulation defect) of the distal radius). 


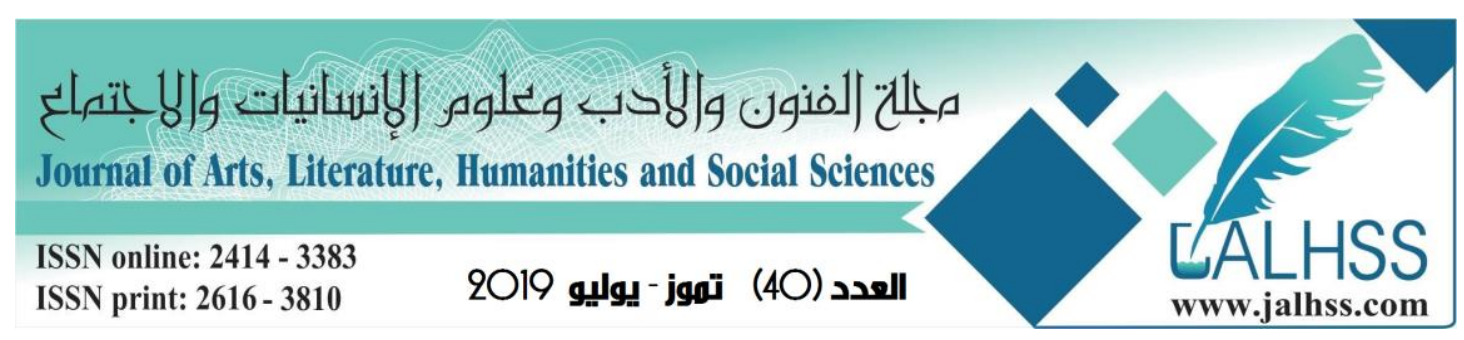

\section{Definition of rickets}

Rickets manifests as a defect in calcification of cartilage matrix at the epiphyseal growth plate due to defective chondrocyte differentia- tion and a delay in mineralization of newly formed osteoid (termed osteomalacia) at the trabecular bone surfaces; and endosteal and peri- osteal surfaces of cortical bone (Pettifor and Thandrayen, 2017). The failure of endochondral calcification results in widening and deforma- tion of the growth plates and subsequent development of visible bony deformities, especially in weight bearing long bones.

\section{What are the risk factors for nutritional rickets?}

The two factors responsible to varying degrees and combinations for the development of nutritional rickets are vitamin D deficiency and dietary calcium deficiency. The vitamin D status of a subject is de- pendent on the amount of vitamin D synthesized in the skin and vitamin D content of the diet. Skin synthesis is dependent on the amount of UV radiation received by the skin, the surface area of skin that is exposed and the degree of melanin pigmentation of the skin. Thus, the risk. factors for vitamin D deficiency and nutritional rickets relate to those factors which reduce sunlight (UV radiation) exposure, such as duration and extent of skin exposure, season of the year, latitude, air-pollution, overcrowding, and increased skin pigmentation, and those factors in- fluencing the dietary intake of vitamin D, such as whether or not foods are vitamin $\mathrm{D}$ fortified and their natural vitamin $\mathrm{D}$ content. Breastmilk normally contains only small amounts of vitamin D (40-60 IU/L) unless the mother is supplemented with high doses of vitamin $\mathrm{D}$, thus all breastfed infants should be vitamin $\mathrm{D}$ supplemented . Further a neonate's vitamin D status is dependent on that of the mother's at the time of delivery, thus babies born to vitamin $\mathrm{D}$ deficient mothers are deficient as well (Wall et al., 2016: 124). The latitude of the area of residence is also a major factor in determining the vitamin D status of un- supplemented children, especially during the winter months, as those residing at latitudes $>34^{\circ} \mathrm{N}$ or S have limited cutaneous vitamin D synthesis due to the negligible amounts of UV radiation reaching the earth during this period. Thus globally the two main causes of vitamin D deficiency are due to geographical and cultural factors, which limit skin exposure to UVB.

In comparison to vitamin D deficiency, the risk factors for dietary calcium deficiency rickets are poor dietary calcium intakes, and diets rich in high phytate cereals, or green leafy vegetables rich in calcium inhibitors such as oxalates. In lower socioeconomic communities, the high cost and relative unavailability of dairy products limits dairy product consumption in the child who has been weaned. Lactose intolerance is not thought to be an important deterrent to milk intake for African and Asian children, as it is infrequent in the pediatric age range, however it can be a cause for low dietary calcium intake. There are no studies investigating the association between lactose intolerance and calcium intake in children presenting with rickets and this aspect requires further investigation. Low-lactose diets result in low dietary 


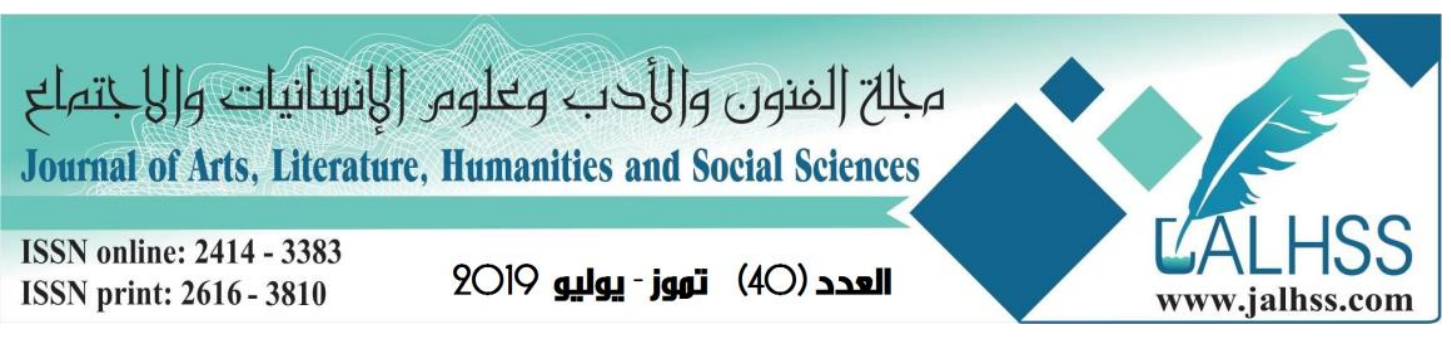

calcium in- takes and low bone mineral content in pre-pubertal children with lac- toseintolerance and lactose intolerance has been suggested to prevent the achievement of an adequate peak bone mass in young adults (Marwaha et al., 2005; Pettifor, 2014: 56) .

Table 1

Biochemistry and treatment administered to patient over the first 12 months.

\begin{tabular}{|l|c|c|c|c|}
\hline \multicolumn{1}{|c|}{ Clinic visits/follow-ups } & Initial presentation & $\begin{array}{c}\text { 3 month } \\
\text { follow-up }\end{array}$ & $\begin{array}{c}\text { 7-8 month } \\
\text { follow-up }\end{array}$ & $\begin{array}{c}\text { 10 month } \\
\text { follow-up }\end{array}$ \\
\hline Serum biochemistry & & & & \\
\hline Calcium (mmol/L) & 1.86 & 2.25 & 2.6 & 2.3 \\
\hline Phosphate (mmol/L) & 1.29 & 1.07 & 1.38 & 1.45 \\
\hline $\begin{array}{l}\text { Alkaline phosphatase } \\
\text { (U/L) (N < 350 IU/L) }\end{array}$ & 691 & 396 & 473 & 363 \\
\hline $\begin{array}{l}\text { Parathyroid hormone (N } \\
1.6-6.9 \text { pmol/L) }\end{array}$ & 21.5 & 4 & 6 & 6.1 \\
\hline $\begin{array}{l}\text { 25 hydroxyvitamin D } \\
\text { (nmol/L) }\end{array}$ & 28.2 & & 49.0 & \\
\hline $\begin{array}{l}1.25 \text { dihydroxyvitamin D } \\
\text { (N = 43-168 pmol/L) }\end{array}$ & 379 & & 404 & \\
\hline $\begin{array}{l}\text { Urine calcium/creatinine ratio } \\
\text { (mmol/mmol) }\end{array}$ & & & 0.14 & \\
\hline TREATMENT & 5000 IU & $2500 \mathrm{IU}$ & - & - \\
\hline Vitamin D (calciferol) & $\begin{array}{l}500 \mathrm{mg} \text { (despite } 1000 \\
\text { mg being prescribed) }\end{array}$ & $1000 \mathrm{mg}$ & $1000 \mathrm{mg}$ & $1000 \mathrm{mg}$ \\
\hline Calcium carbonate & & & & \\
\hline
\end{tabular}

More recent studies in Indian, Mongolian and European children have proposed that nutritional rickets is secondary to both low dietary calcium intake and a low or borderline vitamin D nutritional status (Aggarwal et al., 2016; 34). In an Indian study of children with rickets, 25(OH)D levels were $\leq 20 \mathrm{ng} / \mathrm{mL} \quad(50 \mathrm{nmol} / \mathrm{L})$ but not significantly different from controls. However mean calcium intakes in the rachitic children were approximately half that of controls and similar to levels found in and iraq children $(\approx 200 \mathrm{mg} /$ day $)$. Calcium intakes but not $25(\mathrm{OH}) \mathrm{D}$ levels were inversely associated with the severity of rickets and parathyroid hormone levels. In a European study, Moldovan children with low dietary calcium intakes in combination with low $25(\mathrm{OH}) \mathrm{D}$ levels were associated with a high prevalence of leg deformities, raised alkaline phosphatase levels and low serum calcium concentrations .Thus, a combination of associated risk factors can be accountable for the presentation of nutritional rickets in children residing in dif- ferent countries worldwide with diverse cultural backgrounds. 


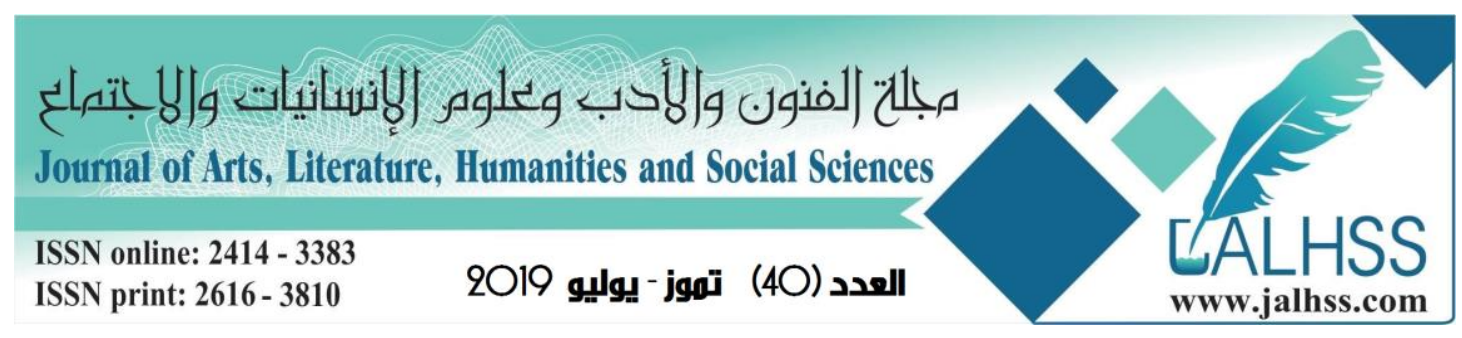

\section{Clinical presentation of nutritional rickets}

The peak prevalence of vitamin D deficiency rickets occurs between 6 months and 2 years of age, when the infant is being breastfed and yet to become independently ambulatory. In some communities, the pre- valence peaks again during late childhood and adolescence probably due to the increased growth rate and social customs, which prevent adequate skin exposure to sunlight. Rickets related to dietary calcium deficiency tends to occur later than vitamin D deficiency. In South Africa, the age of affected children varied between 4 and 16 years, while in Nigeria the average age at presentation was approximately 4 years.

The clinical presentation of nutritional rickets is dependent on the age of the child (Table 2). In the first 6 months of life, symptoms and signs frequently relate to hypocalcaemia (seizures, apneic attacks, stridor and tetany) as a consequence of vitamin D deficiency. The other clinical findings in infants include delayed closure of fontanelles, cra- niotabes and delayed dentition. In older infants and toddlers, the most common clinical findings are widened wrists, rachitic rosary and frontal bossing (Agaba et al., 2016: 78)

Table 2

\section{Age-related changes of the clinical features of rickets}

Age $\quad$ Clinical presentation

$<6$ months Hypocalcaemia

Craniotabes

> 6 months More common:

Chest abnormalities

Enlargement of wrists

Bony deformities

Deformities of the distal radius and ulna Anterior bowing of the tibia

> 1 year On weight bearing:

Deformities of the lower limbs with initially bowing of the legs

But as child gets older, knock-knees and wind-swept deformities At any age Severe rickets can present with:

Fractures

Hypotonia

Sweating

Pain on mobilizing the limbs 


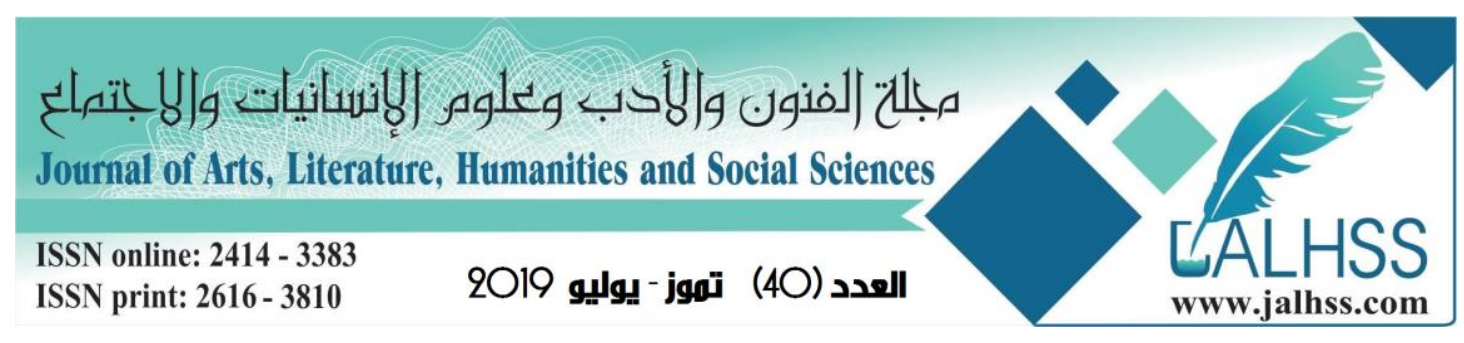

Other features include frontal bossing and macrocephaly in toddlers and Harrison's sulcus and other chest wall deformities in severe rickets. Further hypotonia, excessive sweating and bone tenderness may be present in severely affected infants. In Nigerian children with rickets (older than 18 months of age), costochondral and wrist enlargement were the two clinically independent features with the highest positive predictive value for active rickets. In older children and adolescents, < 50\% of patients with rickets present with lower limb deformities (Voloc et al., 2010). Genu varum tends to pre- sent in infants with rickets when they start to weight-bear or walk. Knock-knees (genu valgum) and wind-swept deformities tend to man- ifest in older children as exemplified in our case report and are more common in children with phosphopenic rickets (Agaba et al., 2016: 62). In adolescents, proximal muscle weakness, presenting as difficulty rising from sitting, may be apparent. In severe nutritional rickets, fractures, especially of the long bones, may occur with minimal trauma.

Our index patient presented with features that are in keeping with less severe rickets or possibly healing rickets. In addition, his radiological findings (described below) were also more in keeping with healing rickets and thus the initial clinical diagnosis by the referring orthopedic surgeon was that of a skeletal dysplasia.

\section{Radiological confirmation of rickets}

The earliest radiographic sign of rickets in young infants is a generalized demineralization of the skeleton, but its detection is rather subjective depending on the quality of the radiographs. Early rickets is manifest as a loss of the provisional zone of calcification of its meta- physeophysis border (seen best at the wrist, the distal femur and proximal tibial metaphysis). More severe signs of active rickets are cupping, fraying or splaying of the metaphyseal ends of the long bones together with increased longitudinal width of the growth plates (normal distance $=1 \mathrm{~mm}$ ) at the wrists and knees. The 10-point Thacher score (radiographic severity score) is a reliable scoring system to assess the severity of active rickets and the improvement in radiological findings while patients are on treatment. The Thacher score was initially validated in children with dietary calcium deficiency rickets over the age of 12 months, however more recently it has been validated for nutritional rickets by Chatterjee et al. (2014). When using the Thatcher score, Chatterjee et al. found that the distal femur is a better indicator of healing in radiologically severe nutritional rickets and when resolution is delayed, it is the last bone to heal (Chatterjee et al., 2014: 43). In our index case, the distal femur still had radiographic changes of active rickets at the metaphysis, supporting the findings of Chatterjee et al. (2014) as the other bones appeared to show sclerotic metaphysical bands indicative of healing.

\section{Pathophysiology of nutritional rickets}




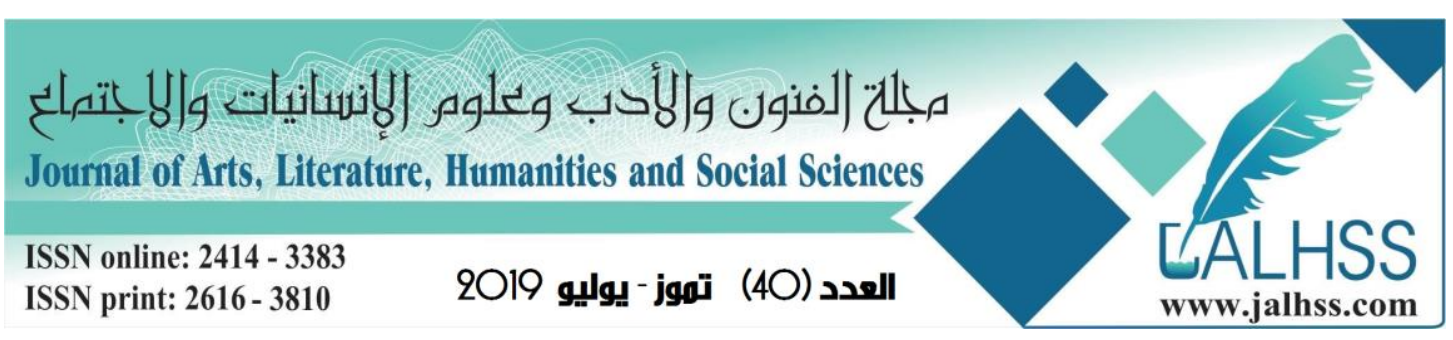

The important question when assessing cases of nutritional rickets is: "What roles do vitamin D deficiency and/or low dietary calcium intakes play in the pathogenesis of the disease?

The case report highlights the presentation of calcium deficiency rickets with concomitant vitamin D deficiency in a young and abundant sunshine throughout the year (an average of nearly $9 \mathrm{~h}$ /day of sunshine over the year). Despite the copious sun- light, it is likely that the index case suffered from vitamin D deficiency while he was cloistered in the children's home, which manifested as a delay in motor milestones. Once he was reunited with his family, it is likely that a poor dietary calcium intake became a more prominent factor in maintaining or aggravating his bone disease. Although dietary calcium or vitamin D deficiency (Al-Mustafa et al., 2007: 82) may in- dividually be responsible for the pathogenesis of nutritional rickets, Results in bold are "abnormal results, out of the normal reference range".

recent studies (Aggarwal et al., 2016: 56) have indicated that both dietary calcium and vitamin D defi- ciencies probably play synergistic roles in the pathogenesis of nutritional rickets in children after infancy, when breastfeeding is un- common and dairy product intake small.

According to the Institute of Medicine (IOM) guidelines and the recent global consensus recommendations on prevention and management of nutritional rickets, vitamin $\mathrm{D}$ deficiency is regarded to be present at $25(\mathrm{OH}) \mathrm{D}$ levels $<30 \mathrm{nmol} / \mathrm{L}$, insufficiency at between 30 and $50 \mathrm{nmol} / \mathrm{L}$ and sufficiency $>50 \mathrm{nmol} / \mathrm{L}$ (Munns et al., 2016: 147). In classical vitamin D deficiency, the risk of developing nutritional rickets with abnormalities of skeletal mineralization and mineral ion metabolism, increases when serum levels of $25(\mathrm{OH}) \mathrm{D}$ fall below $30 \mathrm{nmol} / \mathrm{L}$. Several studies have also confirmed that in the face of normal $25(\mathrm{OH}) \mathrm{D}$ levels, nutritional rickets can present as a result of inadequate dietary calcium intake . A dietary calcium intake of $<300$ $\mathrm{mg} /$ day increased the risk of nutritional rickets independent of $25(\mathrm{OH}) \mathrm{D}$ levels. The global consensus guidelines on prevention and management of nutritional rickets for children over 12 months of age, has classified dietary calcium intake into three categories: sufficiency $>500 \mathrm{mg} /$ day, insufficiency between 300 and $500 \mathrm{mg} /$ day and deficiency $<300 \mathrm{mg} /$ day . Nutritional rickets does not occur in the presence of vitamin D sufficiency and calcium intakes of $>500 \mathrm{mg} /$ day .

Attempts have been made to define vitamin D deficiency based on the 25(OH)D level at which PTH concentrations starts to rise. There are, however, several draw backs to this method: 1) the variation in the association between PTH and $25(\mathrm{OH}) \mathrm{D}$ is large thus making it difficult to detect an inflection point and 2) the relationship is influenced by dietary calcium intakes and requirements (Patel et al., 2016: 58). Many studies conducted in adults and adolescents have shown an inverse relationship between 25(OH)D concentrations and PTH, however the actual point of inflection at which PTH starts to rise is a point of considerable debate. In children results have been similarly variable; a study of children with vitamin D insufficiency $(<50$ $\mathrm{nmol} / \mathrm{L})$ in Northern Spain showed no relationship between $25(\mathrm{OH}) \mathrm{D}$ and PTH 


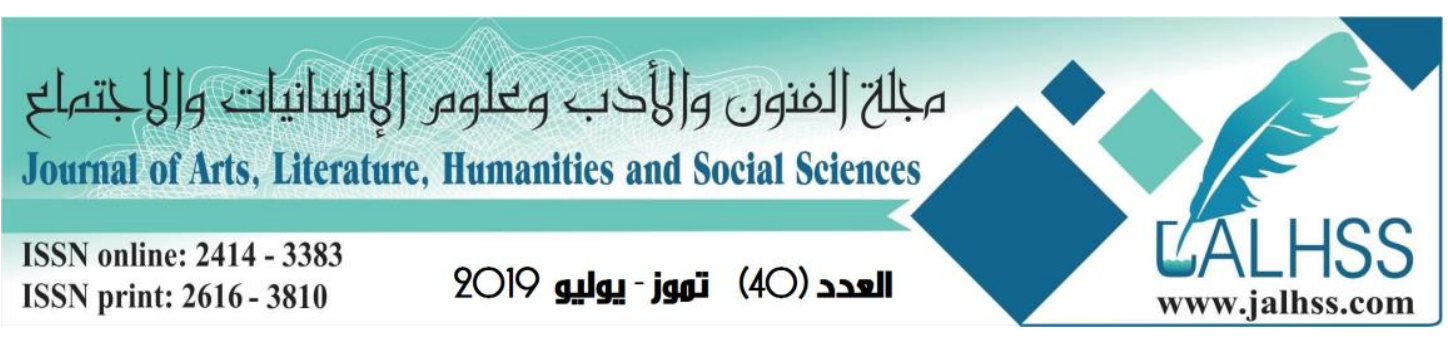

concentrations (Alonso et al., 2015: 90) while in another study by Atapattu et al. (2013), 25(OH)D levels $<34 \mathrm{nmol} / \mathrm{L}$ were associated with an increased release of PTH. In another study of Chinese adolescents who had poor vitamin D status and low calcium intakes, 25(OH)D thresholds were demonstrated at which bone mineralization and turnover were affected (Wu et al., 2015). Below these thresholds (in boys at 33-39 nmol/L and in girls at 20-37 nmol/L), 25(OH)D levels were positively associated with total BMD, and in- aversely associated with serum PTH and TRAP5b; whereas above these thresholds no relationships were found. The influence of dietary calcium intake on the relationship between serum $25(\mathrm{OH}) \mathrm{D}$ concentrations and PTH levels was shown in healthy Indian adolescents, in whom those with higher than median dietary calcium intakes but with similar $25(\mathrm{OH}) \mathrm{D}$ concentrations to the control group had lower PTH concentrations while those with lower dietary calcium in- take had higher PTH concentrations (Patel et al., 2016: 159). Similarly, Djennana et al. emphasized the significant influence of a low calcium diet on the increase in PTH concentrations in children with $25(\mathrm{OH}) \mathrm{D}$ levels $>50 \mathrm{nmol} / \mathrm{L}$. Similarly Aggarwal et al. (2012) reported that in children with similar 25(OH)D levels, those with rickets had very much higher PTH levels than controls, due most likely to associated dietary calcium deficiency in the rachitic children., iraqi children with dietary calcium deficiency rickets have $25(\mathrm{OH}) \mathrm{D}$ levels that are generally low normal. It is suggested that the vitamin $\mathrm{D}$ status is worsened by calcium deficiency itself through the latter inducing secondary hyperparathyroidism and increasing 25(OH)D catabolism (Aggarwal et al., 2016: 87). As suggested by Thacher and Abrams (2010), the proportion of intestinal calcium absorption that is active is greater in calcium deprivation states which increases the requirements for vitamin D.

The salient and characteristic biochemical feature that is indicative of calcium deficiency rather than vitamin $\mathrm{D}$ deficiency is the marked elevation of 1,25dihydroxyvitamin $\mathrm{D}(1,25(\mathrm{OH}) 2 \mathrm{D})$ found prior to treatment, with concentrations being generally 1.5-2.0 times higher than normal controls (Glorieux and Pettifor, 2014: 74). These levels were further increased by nearly 2 fold in Nigerian children with calcium deficiency rickets who were given an oral bolus of vitamin $\mathrm{D}$, an increase not seen in control children (Thacher et al., 2010: 79). Thacher and Abrams (2010) suggest that the increase in $1,25(\mathrm{OH}) 2 \mathrm{D}$ in calcium deficiency rickets implies that vitamin $\mathrm{D}$ status is not optimal, despite elevated baseline $1,25(\mathrm{OH}) 2 \mathrm{D}$ concentrations. This biochemical finding is also clearly shown in our case report (Table 1). PTH is increased in vitamin D deficiency rickets and is usually increased in calcium deficiency rickets. The increased PTH levels in vitamin D deficiency rickets are due to the direct effects of reduced $25(\mathrm{OH}) \mathrm{D}$ production and the subsequent decrease in $1,25(\mathrm{OH}) 2 \mathrm{D}$ concentrations, reducing intestinal calcium absorption and serum calcium concentrations. In calcium deficiency rickets, the inadequate absorption of calcium from the gastrointestinal tract results in an increase in PTH levels that causes an increase in the $1 \alpha$ hydroxylase activity thus increasing levels of $1,25(\mathrm{OH}) 2 \mathrm{D}$; improving the fractional absorption of calcium. 


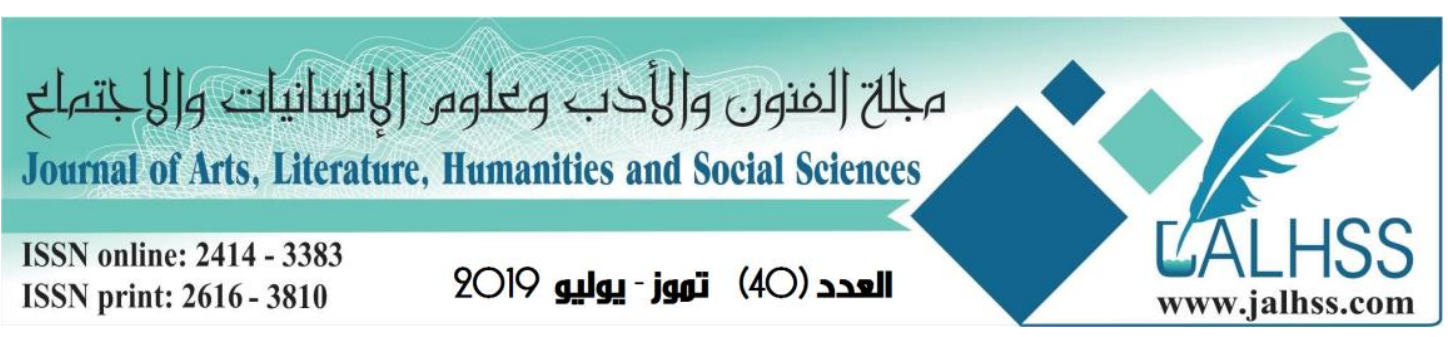

A further feature which distinguishes vitamin D deficiency rickets from that due to dietary calcium deficiency is the difference in fractional intestinal calcium absorption between the two conditions. Studies in Nigeria have found markedly increased fractional calcium absorption (mean 61\%) in children with active rickets due to dietary calcium deficiency prior to treatment (Thacher and Abrams, 2010), which contrasts with the suppressed calcium absorption in vitamin D deficiency. The pathogenesis of vitamin $\mathrm{D}$ and calcium deficiency rickets is shown in Fig. 5. The main role of vitamin $\mathrm{D}$ is to optimize intestinal calcium absorption to meet the needs of the growing child, thus maintaining serum calcium concentrations. It is this role that is thought to be pivotal in the pathogenesis of vitamin $\mathrm{D}$ de- ficiency rickets as inadequate calcium absorption leads to secondary hyperparathyroidism and hypophosphatemia, the latter resulting in inhibition of apoptosis of hypertrophic chondrocytes at the epiphyseal growth plate and impaired endochondral calcification leading to the features of rickets. In dietary calcium deficiency rickets the key mechanism in its pathogenesis is similar to that in vitamin D deficiency - inadequate intestinal calcium absorption to meet the needs of the organism, however an added pathogenetic factor in dietary calcium deficiency is the high $1,25(\mathrm{OH}) 2 \mathrm{D}$ concentrations, which in animal studies have been shown to directly inhibit bone matrix mineralization and redirect skeletal calcium toward the serum; helping to maintaining normocalcaemia when intestinal calcium transport is inadequate, but exacerbating the features of osteomalacia (Lieben et al., 2012: 88).

A unique finding has been reported in children from The Gambia with bone deformities in keeping with dietary calcium deficiency. Serum fibroblast growth factor 23 (FGF23) levels were elevated in a large proportion of the cases, and were inversely associated with plasma haemoglobin concentrations in the 5 year follow-up children with more marked rachitic-like bone deformities. The lower haemoglobin levels in these Gambian children suggest a lower iron status as iron deficiency is endemic in Gambia and the inverse relationship supports the involvement of iron in FGF23 metabolism. Iron is thought to play a role in the clearance of FGF23 fragments by the kidney and to inhibit cleavage of intact FGF23. Increased FGF23 levels are characteristically associated with certain forms of genetic or acquired (tumourinduced osteomalacia with increased production of FGF23) hypophosphataemic rickets. Despite elevated FGF23 levels in the Gambian children1,25 ، (OH)2D levels were increased in response to their low dietary calcium intake and may have contributed to the elevated FGF23 levels (Prentice et al., 2008). Osteoblastic FGF23 expression is stimulated by 1,25 (OH)2D (Kolek et al., 2005: 102). The increase in FGF23 reduces renal phosphate reabsorption through decreased production of sodium- phosphate cotransporters IIa and inhibits $1,25(\mathrm{OH}) 2 \mathrm{D}$ production through down-regulation of $1 \alpha$-hydroxylase. This negative feedback loop maintains phosphate homeostasis and prevents hyperphosphataemic spikes. Whether or not elevated FGF23 levels are found in children with rachitic-like bone disease from other communities requires further study. 


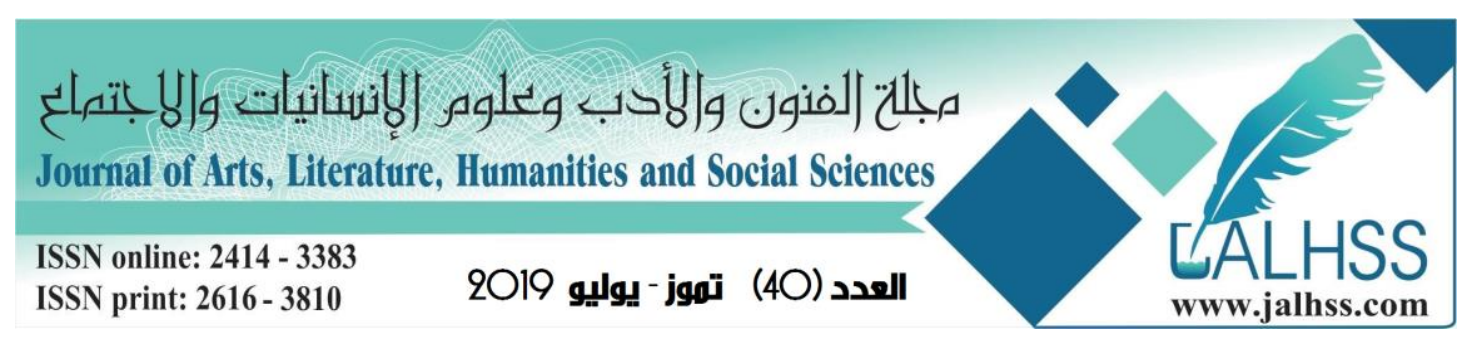

Table 3

Biochemical differences between vitamin D and calcium deficiency rickets.

\begin{tabular}{|l|l|l}
\hline \multicolumn{1}{c}{ Biochemistry Vitamin D deficiency rickets $\quad$ Calcium deficiency rickets } \\
\hline Calcium & $\downarrow / \mathrm{N}$ & $\downarrow \downarrow / \mathrm{N}$ \\
\hline Phosphate & $\mathrm{N} / \downarrow$ & $\mathrm{N} / \downarrow$ \\
\hline Alkaline phosphatase & $\uparrow \uparrow$ & $\uparrow \uparrow$ \\
\hline Parathyroid hormone & $\uparrow$ & $\mathrm{N} / \uparrow$ \\
\hline 25 hydroxyvitamin D & $\downarrow \downarrow(<30 \mathrm{nmol} / \mathrm{L})$ & $\mathrm{N} /$ borderline low \\
\hline 1.25 dihydroxyvitamin D & $\mathrm{N} / \downarrow$ & $\uparrow \uparrow$ \\
\hline
\end{tabular}

Apart from the increase in $1,25(\mathrm{OH}) 2 \mathrm{D}$, which differentiates calcium from vitamin $\mathrm{D}$ deficiency rickets, other biochemical differences and similarities between these two nutritional deficiencies are outlined and summarized in Table 3. In both these deficiencies, serum calcium and phosphate levels are typically low. Secondary hyperparathyroidism is present in both deficiencies as a consequence of hypocalcaemia. Phosphate levels may be normal in the early or mild stages of the disease but as time progresses and secondary hyperparathyroidism develops, hypophosphataemia become progressively more apparent. 25(OH)D levels are very low (frequently $<10-15 \mathrm{nmol} / \mathrm{L}$ ) in vitamin $\mathrm{D}$ deficiency rickets but can be normal or borderline low in calcium deficiency.

\section{Treatment of nutritional rickets}

Preventative strategies are the first and foremost approach to de- creasing the prevalence and occurrence of nutritional rickets especially in high risk communities. Opportunities to provide education on adequate sun-exposure especially in areas with abundant sunshine are mandatory, although concerns about the relationship between sun ex- posure and skin cancers have resulted in recommendations being made that infants should avoid direct sun exposure ("Ultraviolet light: a ha- zard to children. American Academy of Pediatrics. Committee on Environmental Health," 1999) and sunshine exposure should be restricted in older children. The average fair-skinned individual (Fitzpatrick (1988) skin type II) with $1 / 4$ of their skin exposed to sunlight is able to produce 1000 IU vitamin D within 14-30 min while the dark- skinned type IV individual requires 57-58 min or nearly a 2-4 fold longer duration of sun exposure (Gill and Kalia, 2015: 90). Food fortification with vitamin D (mainly staple foods) and routine vitamin $\mathrm{D}$ supplementation are the priorities for preventing vitamin $\mathrm{D}$ 


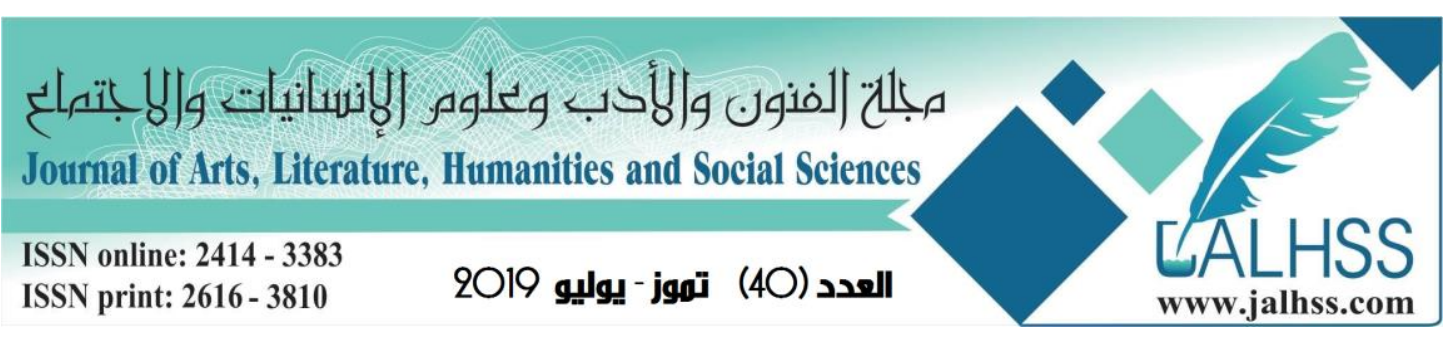

deficiency rickets. Margarine, cereals, cow's milk (in some countries) and milk formulas are fortified but the fortification standards may vary across countries worldwide. The global consensus recommendations on prevention and management of nutritional rickets are that all infants up to 12 months receive oral vitamin D supplementation (400 IU/day) regardless of feeding method or rickets risk and that high risk groups continue supplementation thereafter (Munns et al., 2016: 69). In order to implement routine vitamin D supplementation, it should be part of the routine well-baby clinic visits and possibly be incorporated into the vaccination schedules for infants. The consensus statements also advocated that all pregnant women receive 600 IU/day of vitamin D supplementation together with adequate calcium intakes to prevent congenital rickets (Munns et al., 2016). Lactating mothers should meet the dietary recommendations of $600 \mathrm{IU}$ of vitamin D for their own needs as there is evidence to suggest that there is no extra benefit to the infant from extra vitamin D supplementation to the lactating mother (Munns et al., 2016). Infants of mothers supplemented with $2000 \mathrm{IU} /$ day or more have similar $25(\mathrm{OH}) \mathrm{D}$ levels as infants receiving $400 \mathrm{IU} /$ day and for safety reasons, current recommendations suggest infant supple- mentation rather than high dose maternal supplementation.

Diets rich in dairy products should be recommended to prevent calcium deficiency rickets but this might be difficult in less affluent communities. Lactose intolerance and avoidance of milk in children result in low calcium absorption. Lactose-free and lactose-reduced milk can be substituted for normal formula feeds in lactose intolerant infants but breastfed infants should be continued on human milk. Tolerance to milk products may be partial so small amounts of lactose spaced throughout the day with meals can be tolerated and two glasses of milk may be tolerated without problems .

Despite the simplicity of the prevention and treatment of vitamin D deficiency, many studies have confirmed poor patient treatment adherence, more so in preventative strategies (Grant et al., 2014: 232). The option of stoss therapy or single large dose vitamin D treatment to combat the problems associated with treatment adherence has been recommended. Studies have shown that a single high dose of 300,000-600,000 IU vitamin D2 or D3 given intramuscularly or orally is effective but hypercalcaemia or hypercalciuria as a side effect has been encountered (Mittal et al., 2014; 38). Lower doses of 150,000 or 200,000 IU have been shown to be effective as well without the adverse effects .

Despite the effectiveness of vitamin $\mathrm{D}$, given either as a daily dose or as a bolus, in the management of vitamin D deficiency rickets, several studies have highlighted the need to include calcium supplements to manage hypocalcaemia in severe vitamin D deficiency, in young infants with symptomatic hypocalcaemia and in children with suspected dietary calcium deficiency.

Table 4 provides the vitamin D treatment options (daily and stoss therapy) for nutritional rickets and the supplementation doses following completion of treatment doses (Munns et al., 2016) . 


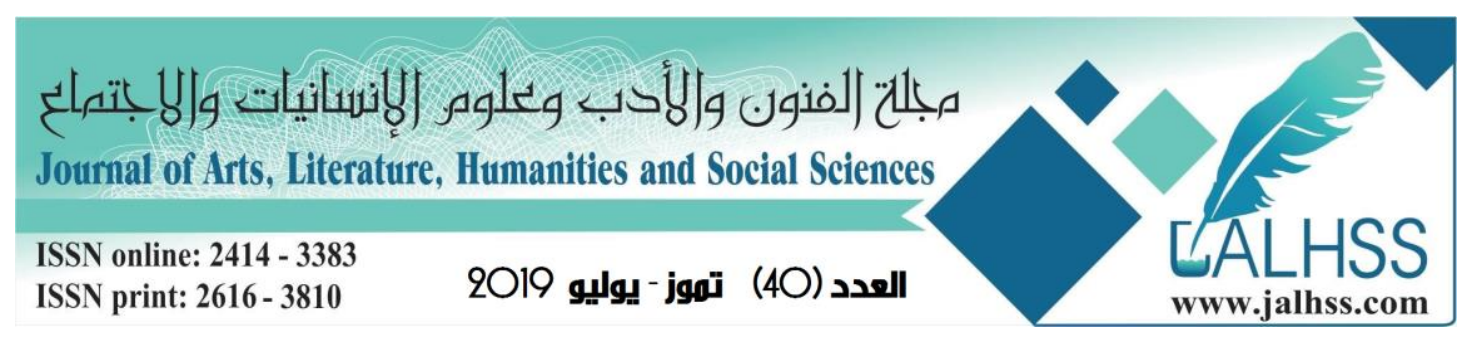

Combined poor vitamin D status and low dietary calcium intakes are becoming recognized more commonly, especially in low and middle income countries. A recent Indian study of children with nutritional rickets found that the participants had a mean dietary calcium intake of $204 \mathrm{mg} /$ day and mean 25(OH)D level of $15.9 \mathrm{ng} / \mathrm{mL}$. These children responded better to a combination of vitamin D $(600,000$ IU single intramuscular injection) and calcium $(75 \mathrm{mg} / \mathrm{kg} /$ day elemental calcium orally) than to calcium alone with $50 \%$ in the former group responding within 12 weeks compared to $15.7 \%$ in calcium alone group (Aggarwal et al., 2016). Similar results were found in a Nigerian study, in which rachitic children on combination therapy (vitamin D and calcium) had a more rapid initial decline in serum alkaline phosphatase and improvement in radiological score compared to those on calcium alone .

Thacher et al. (2014) further investigated the response of rickets in Nigerian children to calcium treatment administered as limestone with and without vitamin D supplementation and found that vitamin D once again facilitated more rapid healing and improved vitamin $\mathrm{D}$ status. The response to treatment was independent of baseline $25(\mathrm{OH}) \mathrm{D}$ concentrations. An additional study in Nigerian children with rickets, comparing treatment with limestone to ground fish, found similar healing effects in the two groups (Thacher et al., 2015). These studies (Thacher et al., 2014; Thacher et al., 2015) recommend that limestone or ground fish mixed with food or porridge (provided the child com- pletes the meal) can be used as inexpensive sources of calcium in low- income countries where low calcium intakes and rickets are prevalent. The reasons why vitamin $\mathrm{D}$ enhances healing of rickets in children with dietary calcium deficiency is unclear. It has been shown that vi- tamin D does not improve the already established maximal fractional calcium absorption in Nigerian children with rickets and that the response to therapy is unrelated to initial $25(\mathrm{OH}) \mathrm{D}$ concentrations (Thacher et al., 2009). As has been reported by Thacher, administration of a bolus of vitamin $\mathrm{D}$ in children with active rickets leads to a rapid nearly two fold elevation in 1,25(OH)2D levels above the already elevated levels prior to treatment. It appears that vitamin D metabolites may have a direct or indirect effect on bone mineralization by mechanisms other than calcium absorption (Anderson et al., 2012: 182). As explained earlier under the pathophysiology of calcium deficiency, Lieben et al. described the effects of high $1,25(\mathrm{OH}) 2 \mathrm{D}$-mediated regulation of the bone mineralization inhibitors whose role it is to maintain normocalcaemia but the authors emphasized that there are other modulators and/or post-translational modifications that cannot be excluded as possible co-mechanisms for bone remodeling . 


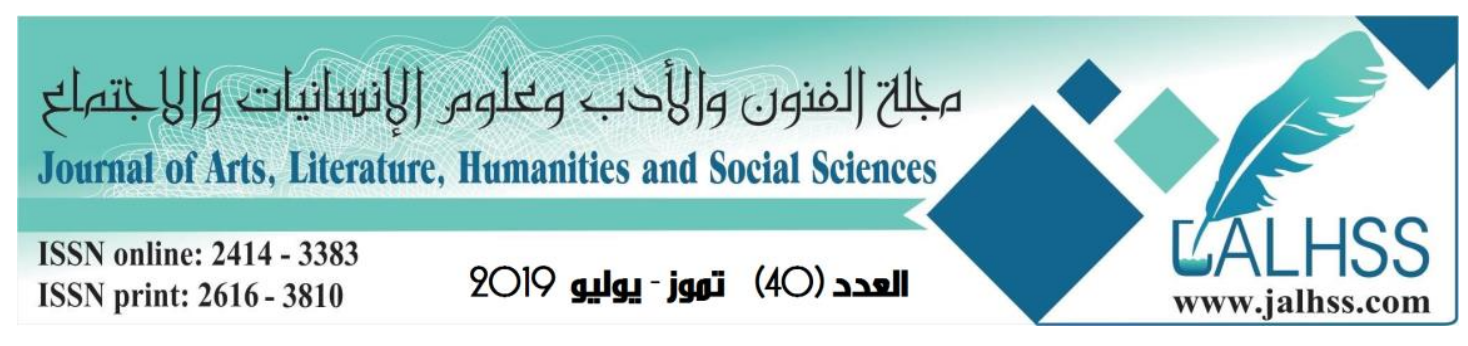

Table 4

Treatment doses of vitamin $D$ for nutritional rickets.

\begin{tabular}{|c|c|c|c|}
\hline Age & Daily dose for & Single dose, & $\begin{array}{c}\text { Maintenance } \\
\text { daily }\end{array}$ \\
\hline & 90 days, IU & IU & dose, IU \\
\hline$<3$ months & 2000 & N/A & 400 \\
\hline $3-12$ months & 2000 & 50,000 & 400 \\
\hline$>12$ months to & $3000-6000$ & 150,000 & 600 \\
\hline$>12$ years & 600 & 300,000 & 6000 \\
\hline
\end{tabular}

Abbreviation: N/A, not available. Reassess response to treatment after 3 months as further treatment may be required. Ensure a daily calcium intake of at least $500 \mathrm{mg}$. For con- version from IU to $\mu \mathrm{g}$, divide by 40 .

What is the optimal dose of calcium for treatment of nutritional rickets secondary to calcium deficiency? The calcium requirements in children with healing rickets may be greater than those of normal children because of "hungry bones". A daily dose of $1000 \mathrm{mg}$ is optimal for treatment of children with a customary dietary calcium intake $<300 \mathrm{mg}$ /day and some children required treatment for > 24 weeks for complete healing of rickets (Thacher et al., 2016). Fractional calcium absorption is greatest with calcium intakes of $<500 \mathrm{mg} /$ day and the total calcium absorption may not be significantly greater with a $2000 \mathrm{mg}$ dose compared with a $1000 \mathrm{mg}$ dose (Thacher et al., 2016) .

The global consensus recommendations on prevention and management of nutritional rickets recommends that oral calcium $(500 \mathrm{mg} /$ day) either as dietary intake or supplements, should be routinely used in conjunction with vitamin D in the treatment regardless of age and weight.

As documented in this case presentation, the patient responded well to at least three months of oral calcium of $500 \mathrm{mg} /$ day and vitamin D of $5000 \mathrm{IU} /$ day (Table 1).

\section{Conclusion}

Nutritional rickets is the most common metabolic bone disorder globally despite its ease of prevention and treatment. A thorough de- tailed history of diet and associated risk factors, together with the clinical features of rickets should lead the attending clinician to the underlying diagnosis, be it calcium deficiency or vitamin D deficiency or a combination of both. The important biochemical clues to the underlying dietary calcium deficiency will be that of a low calcium, high 1,25(OH)2D and concomitant low-normal vitamin D status whereas untreated vitamin D deficiency rickets will have low $25(\mathrm{OH}) \mathrm{D}$ levels usually in the range $<10 \mathrm{nmol} / \mathrm{L}$. In resource constrained settings, the global consensus recommendations for the management and treatment of nutritional rickets should be followed, which recommend the ad- ministration of at 


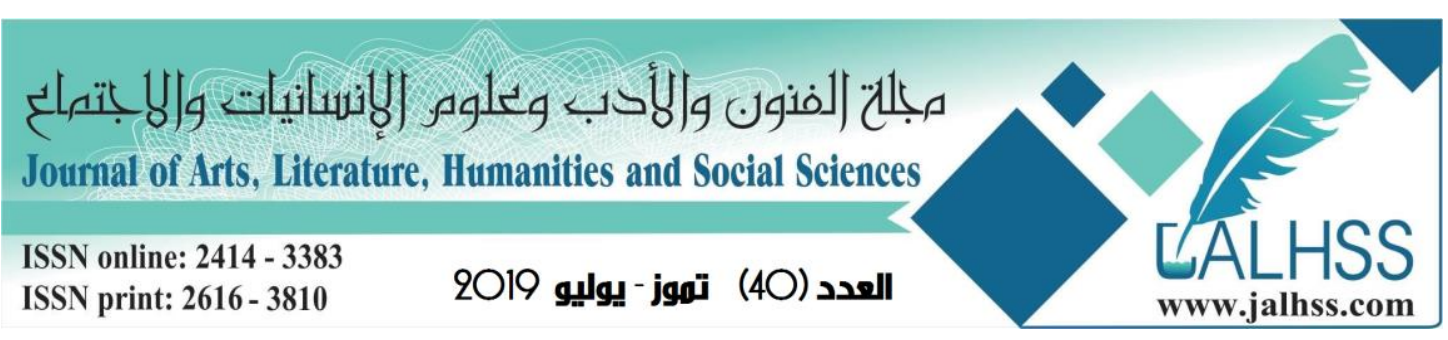

least $500 \mathrm{mg}$ of calcium per day and vitamin D therapy according to age. Response to therapy should be reassessed after 3 months; if clinical, biochemical and radiographic evidence of improvement has occurred, treatment should be maintained for a fur- ther three months, while if no evidence of improvement has occurred, it is imperative that adherence to the treatment regimen be assessed be- fore investigating the rarer often genetic causes of calciopenic rickets further.

\section{References}

1. Agaba, F., Pettifor, J.M., Thandrayen, K., 2016. Comparative study of children with calciopenic and phosphopenic rickets seen at Chris Hani Baragwanath Hospital. SA Orthop. J. 15, 37-42.

2. Aggarwal, V., Seth, A., Marwaha, R.K., Sharma, B., Sonkar, P., Singh, S., Aneja, S., 2016. Management of nutritional rickets in Indian children: a randomized controlled trial.

3. Al-Mustafa, Z.H., Al-Madan, M., Al-Majid, H.J., Al-Muslem, S., Al-Ateeq, S., AlAli, A.K., 2007. Vitamin D deficiency and rickets in the Eastern Province of Saudi Arabia. Ann. Trop. Paediatr. http://dx.doi.org/10.1179/146532807x170529.

4. Anderson, P.H., Turner, A.G., Morris, H.A., 2012. Vitamin D actions to regulate calcium and skeletal homeostasis. Clin. Biochem. 45, 880-886. http://dx.doi.org/10.1016/j. clinbiochem.2012.02.020.

5. Chatterjee, D., Gupta, V., Sharma, V., Sinha, B., Samanta, S., 2014. A reliable and cost effective approach for radiographic monitoring in nutritional rickets. Br. J. Radiol. 87, 20130648. http://dx.doi.org/10.1259/bjr.20130648.

6. Gill, P., Kalia, S., 2015. Assessment of the feasibility of using sunlight exposure to obtain the recommended level of vitamin D in Canada. C. Open 3, E258-63. http://dx.doi. org/10.9778/cmajo.20140037.

7. Glorieux, F.H., Pettifor, J.M., 2014. Vitamin D/dietary calcium deficiency rickets and pseudo-vitamin D deficiency rickets. Bonekey Rep. 3, 524. http://dx.doi.org/10. 1038/bonekey.2014.19.

8. Grant, C.C., Stewart, A.W., Scragg, R., Milne, T., Rowden, J., Ekeroma, A., Wall, C., Mitchell, E.A., Crengle, S., Trenholme, A., Crane, J., Camargo Jr., C.A., 2014. Vitamin D during pregnancy and infancy and infant serum 25-hydroxyvitamin D concentra- tion. Pediatrics 133, e143-53. http://dx.doi.org/10.1542/peds.2013-2602.

9. Lieben, L., Masuyama, R., Torrekens, S., Van Looveren, R., Schrooten, J., Baatsen, P., Lafage-Proust, M.H., Dresselaers, T., Feng, J.Q., Bonewald, L.F., Meyer, M.B., Pike, J.W., Bouillon, R., Carmeliet, G., 2012. Normocalcemia is maintained in mice under conditions of calcium malabsorption by vitamin D-induced inhibition of bone mi- neralization. J. Clin. Invest. 122, 1803-1815. http://dx.doi.org/10.1172/jci45890.

10. Marwaha, R.K., Tandon, N., Reddy, D.R., Aggarwal, R., Singh, R., Sawhney, R.C., Saluja, B., Ganie, M.A., Singh, S., 2005. Vitamin D and bone mineral density status of healthy schoolchildren in northern India. Am. J. Clin. Nutr. 82, 477-482. 


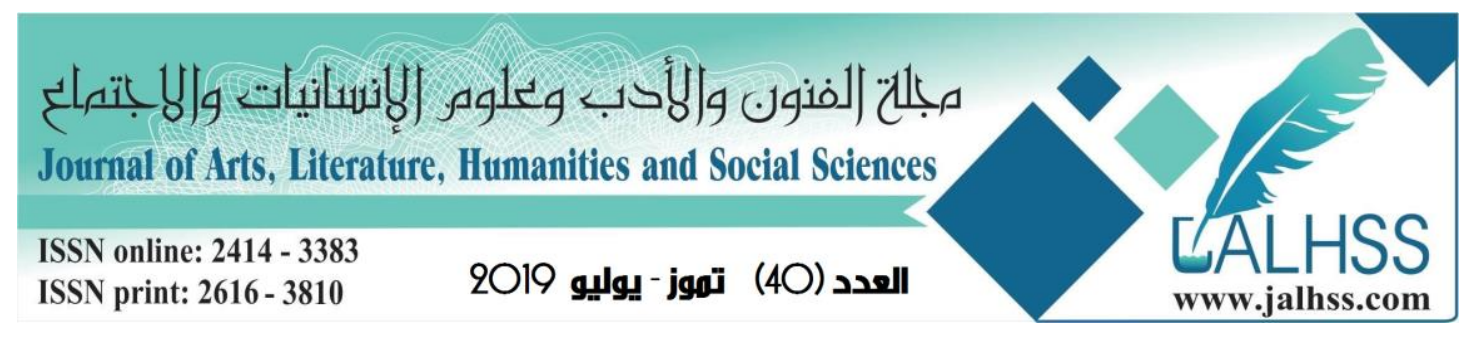

11. Mittal, H., Rai, S., Shah, D., Madhu, S.V., Mehrotra, G., Malhotra, R.K., Gupta, P., 2014. 300,000 IU or 600,000 IU of oral vitamin D3 for treatment of nutritional rickets: a randomized controlled trial. Indian Pediatr. 51, 265-272.

12. Munns, C.F., Shaw, N., Kiely, M., Specker, B.L., Thacher, T.D., Ozono, K., Michigami, T., Tiosano, D., Mughal, M.Z., Makitie, O., Ramos-Abad, L., Ward, L., DiMeglio, L.A., Atapattu, N., Cassinelli, H., Braegger, C., Pettifor, J.M., Seth, A., Idris, H.W., Bhatia, V., Fu, J., Goldberg, G., Savendahl, L., Khadgawat, R., Pludowski, P., Maddock, J., Hypponen, E., Oduwole, A., Frew, E., Aguiar, M., Tulchinsky, T., Butler, G., Hogler, W., 2016. Global consensus recommendations on prevention and management of nutritional rickets. J. Clin. Endocrinol. Metab. 101, 394-415. http://dx.doi.org/10. 1210/jc.2015-2175.

13. Patel, P., Mughal, M.Z., Patel, P., Yagnik, B., Kajale, N., Mandlik, R., Khadilkar, V., Chiplonkar, S.A., Phanse, S., Patwardhan, V., Patel, A., Khadilkar, A., 2016. Dietary calcium intake influences the relationship between serum 25-hydroxyvitamin D3 (25OHD) concentration and parathyroid hormone (PTH) concentration. Arch. Dis. Child. 101, 316-319. http://dx.doi.org/10.1136/archdischild-2015-308985.

14. Pettifor, J.M., Thandrayen, K., 2017. Nutritional rickets and vitamin D deficiency. In: de Pee, S., Taren, D., Bloem, M.W. (Eds.), Nutrition and Health in a Developing World. Springer Science+Business Media, New York, pp. 297-319.

15. Thacher, T.D., Fischer, P.R., Obadofin, M.O., Levine, M.A., Singh, R.J., Pettifor, J.M., 2010. Comparison of metabolism of vitamins D2 and D3 in children with nutritional rickets. J. Bone Miner. Res. 25, 1988-1995. http://dx.doi.org/10.1002/jbmr.99.

16. Voloc, A., Esterle, L., Nguyen, T.M., Walrant-Debray, O., Colofitchi, A., Jehan, F., Garabedian, M., 2010. High prevalence of genu varum/valgum in European children with low vitamin D status and insufficient dairy products/calcium intakes. Eur. J. Endocrinol. 163, 811-817. http://dx.doi.org/10.1530/eje-10-0434.

17.Wall, C.R., Stewart, A.W., Camargo Jr., C.A., Scragg, R., Mitchell, E.A., Ekeroma, A., Crane, J., Milne, T., Rowden, J., Horst, R., Grant, C.C., 2016. Vitamin $\mathrm{D}$ activity of breast milk in women randomly assigned to vitamin D3 supplementation during pregnancy. Am. J. Clin. Nutr. 103, 382-388. 